\title{
Mechanical Property, Thermal Stability and Bio- Degradability Studies on PLA based Bio-Nanocomposites derived from Agricultural Residues
}

\author{
Mrudhula Reddivari ${ }^{1 *}$ and Dr. P. Dinesh Sankar Reddy ${ }^{2}$ \\ ${ }^{1}$ Department of Chemical Engineering, JNTUA Anantapuramu -515002 \\ ${ }^{2}$ National Institute of Technology Andhra Pradesh, Tadepalligudem-534101 \\ ${ }^{1}$ mrudhulabalam@gmail.com, ${ }^{2}$ pdsreddy@gmail.com
}

\begin{abstract}
Looking at the environmental hazards being posed by indiscriminate use of synthetic plastics, abundant research is being done to explore various bio-degradable polymers. In the present study, Cellulose Nano Fibers (CNFs) were extracted from Pineapple Crown using mechano chemical treatment, PLA was synthesized by Simultaneous Saccharification and Fermentation using cellulase enzyme on Acacia Arabica as substrate. Further, ZnO nanoparticles were synthesized by using different precursors. The biocomposite sheets of PLA, PLA+ 5\%-20\% CNFs, PLA+5\% ZnO+5-20\% CNFs and PLA+10\% ZnO+5-20\% CNFs were solvent casted. Microbial efficacy test was done using E.coli and with inclusion of $\mathrm{ZnO}$ nanoparticles the microbial resistance has increased. Noteworthy vibration band of the sheets were observed in the wavelength range of 3700 to $2800 \mathrm{~cm}^{-1}$ from the FTIR analysis, which shows that there is only a physical interaction rather than chemical. The crystallinity increased for initial concentration, but was similar to the neat PLA. Significant increase in tensile strength and maximum elongation at break was observed in PLA $+5 \% \mathrm{ZnO}+10 \%$ CNFs sheet. Sheets were allowed to degrade naturally and significant weight loss was observed after 120 days with maximum reduction of $38.4 \%$. Morphological analysis through SEM revealed the uniform distribution of fillers in the polymer matrix. TGA studies have shown that the degradation temperatures were in the range of $320-405^{\circ} \mathrm{C}$. The thermal stability decreased with the increase in $\mathrm{ZnO}$ concentration. The results have shown a promising and sustainable use in various applications in view of microbial resistance and bio-degradability.
\end{abstract}

Key words: Cellulose Nano Fibres; Poly Lactic Acid; Biodegradability; Antimicrobial;

\section{Introduction}

Environmental issues caused by petroleum polymers have shifted the focus of research on to the bio-polymers in the past two decades. Polymers derived from the monomeric molecules from natural resources or living organisms are known as bio-polymers. Bio-polymers which are abundant in nature have advantages like bio-degradability, 
renewability, co-efficiency and they also have disadvantages like moisture absorption, lesser mechanical properties and cost effectiveness. To overcome these disadvantages, the focus has been shifted to the development of bio-nano-composites.

Composites, consists of two different materials with discrete properties combined to form a continuous matrix and reinforcement as discontinued phase. Matrix phase generally consists of thermoset or thermoplastic resins which are derived from the petroleum products. Fillers are added to tailor the properties of the polymers. But now emphasis has been shifted to the bio based polymer composites. Biocomposite systems can be classified into complete biodegradable and partially biodegradable biocomposites on the basis of the matrix and fibers $[4,5]$. In the present study, complete biodegradable of the biocomposites have been chosen.

Poly Lactic Acid is a linear aliphatic thermoplastic polymer which is produced from the renewable natural resources by fermentation of poly saccharides or sugar. It's a hydrophobic biodegradable polymer with a basic monomer of lactic acid $\left[\left(\mathrm{C}_{3} \mathrm{H}_{4} \mathrm{O}_{2}\right) n\right]$. PLA exhibits many properties that are equivalent to or better than many petroleum based plastics which makes it suitable for a variety of applications. PLA is a versatile polymer that has many potential uses, including many applications in the textile and medical industries as well as the packaging industries. PLA polymer can be amorphous, semi-crystalline, and highly crystalline polymer depending on the stereoisomer composition. PLA's mechanical properties depend on various parameters, e.g., component isomers, processing temperature, annealing time, and molecular weight $(\mathrm{Mw})$. Generally, it has high strength and moderate barrier properties. Simultaneously, it is relatively less thermally stable, decomposing below $230^{\circ} \mathrm{C}$ and has a poor toughness with a low deformation at the break. For example, semicrystalline PLA, which is preferred over the amorphous PLA from the mechanical properties point of view, has an approximate tensile modulus of $3 \mathrm{GPa}$, a tensile strength of 50-70 $\mathrm{MPa}$, and an elongation at break of about $4 \%[6,8,10]$.

Cellulose is a widely available natural polymer which can be obtained from various plant sources, animal sources and from bacterial sources. Cellulose from various parts of the plant - like stem, leaf, seeds and bast are being researched extensively as they show varied properties depending on their source. Advances in Nano Science has also focused on the nanocellulose produced from various sources of plants and animals. Due to its versatile properties, they are conventionally used as reinforcements in many polymer composites. Pineapple is one of the tropical fruit crop grown in north eastern states of India. An adult Pineapple plant bears approximately 80 leaves per plant, where only very small portions are being used in the field of feed stock and energy production. With progress in research on utilization of pineapple leaves, Indian Council for Agriculture Research has started a pilot project to extract pineapple leaf fibres used in textile industry, as the leaves contain $2.5-3.5 \%$ good fibre. The main chemical constituents of pineapple fibres are cellulose (70-82\%), lignin $(5-12 \%)$ and ash $(1.1 \%)$. The superior mechanical properties of pineapple leaf fibres are associated with their high cellulose content.[3]

Among the various types of Nano particles derived from metals and metal oxides, Nano Zinc oxide has got a special focus due its versatile properties. Nano $\mathrm{ZnO}$ has prominent 
physical and chemical properties like high luminous intensity, intensive UV absorption, antimicrobial activity, high band gap etc.

In the present study nano cellulose fibers extracted from pineapple crown has been reinforced into PLA polymer matrix. Hybrid composites have been solvent casted by adding nano- $\mathrm{ZnO}$ at various concentrations along with PLA and nanocellulose from pineapple leaf fibres. The morphology, crystallinity and thermal properties of the isolated nanofibres were characterized by Scanning Electron Microscope (SEM), X-Ray Diffraction (XRD), ThermoGravimetric Analysis (TGA) and DSC. Further the mechanical properties, biodegradability and microbial resistance have been studied.

\section{Experimental Work}

\subsection{Materials:}

Poly Lactic Acid was synthesized by Simultaneous Sacharification and Fermentation using cellulase enzyme on Acacia Arabica as substrate and Cellulose Nano Fibers (CNFs) were extracted from Pineapple Crown leaves using mechano chemical treatment. Further, $\mathrm{ZnO}$ nanoparticles were synthesized by using different precursors. Dichloromethane was used as a solvent. All the chemicals used were of analytical grade without further purification.

\subsection{Preparation of PLA and PLA composite films:}

PLA based nano composites were prepared using solution casting method. In brief, PLA and Dichloromethane are dissolved in the ratio of 1:6 in a MK-GB1 stand mixer for 3 hours at a speed of $600 \mathrm{rpm}$ at room temperature. The composite films were obtained by mixing nano particles into the dissolved solution and stirred for one hour in the same mixer. The dissolved composite film is poured on to the plain glass sheet and dried at the ambient temperature for 24 hours. The biocomposite sheets of PLA, PLA+ 5\%-20\% CNFs, PLA+5\% $\mathrm{ZnO}+5-20 \% \mathrm{CNFs}$ and PLA+10\% $\mathrm{ZnO}+5-20 \% \mathrm{CNFs}$ were solvent casted.

\subsection{Scanning Electron Microscopic analysis:}

The morphology of each sheet was examined under a Zeiss DSM 960 microscope with a scanning voltage of $10 \mathrm{kV}$.

\subsection{Crystallinity measurement:}

XRD pattern of bio-composite samples was recorded over a $2 \theta$ range of $10-35^{\circ}$ using Bruker D8 Advance, Germany with $\mathrm{Cu} K \alpha$ radiation $(\lambda=1.54 \AA)$ at a scan speed $0.04^{\circ} \mathrm{s}^{-1}$.

\subsection{Fourier Transform Infrared Spectroscopy (FTIR):}

FTIR spectroscopy was done using Bruker - FTIR device - ALPHA Eco - ATR. The samples' spectra were recorded using Opus software at 32 scans, with a resolution of $4 \mathrm{~cm}^{-1}$, within the wave number range of 4000 to $400 \mathrm{~cm}^{-1}$. The "find peak tool", provided by Opus software, was used to measure the significant transmittance peaks at particular wave numbers. 


\subsection{Thermal analysis:}

The thermal stability was determined using a thermo gravimetric analyzer (Perkin Elmer, Diamond) and the temparature range was $25^{\circ} \mathrm{C}-700^{\circ} \mathrm{C}$ with a heating rate of $10^{\circ}$ $\mathrm{C} / \mathrm{min}$. The thermal stability, melting and crystalline temperature of composites were analyzed in thermo gravimetric analysis (TGA) and Differential Scanning Calorimetry (DSC). The heating rate was $10^{\circ} \mathrm{C} / \mathrm{min}$ was maintained in both DSC and TGA analysis.

\subsection{Mechanical Properties:}

Mechanical tests i.e., uni axial tensile responses of the composites were measured using Universal Testing Machine (UTM) Zwick Z250. The load cell was mentioned 10KN for testing all polymeric samples at a speed of $5 \mathrm{~mm} / \mathrm{min}$. The specimens of composite sheets were tested with a sample dimension of $\sim 80 \mathrm{~mm} \times 20 \mathrm{~mm} \times 1 \mathrm{~mm}$. To ensure the repeatability and uniformity of testing at-least five samples of each composition were tested and the average was calculated. Tensile strength, Young's Modulus and elongation at break of all the composites were measured.

\subsection{Bio-degradability Test:}

To know the extent of biodegradability of the films prepared, the films have been cut into the size of $5 \times 5 \mathrm{~cm}$ and dried in a desiccator until the moisture is removed and the weight becomes constant. The samples were then buried in the glass containing the garden soil for a period of 120 days. The initial weight of the samples was measured [ $\mathrm{W}_{1}$ ] so that the loss of weight at periodical intervals can be compared. The samples were taken out after 10, 30, 60 and 120 days for weight measurement. The samples were cleaned properly to remove the soil residue so as to obtain accurate weight of the samples $\left[\mathrm{W}_{2}\right]$. The percentage of weight loss $(\% \mathrm{WL})$ was calculated.

\subsection{Microbial resistance:}

A loop-full of culture was inoculated form culture bank and inoculated to $10 \mathrm{ml}$ of nutrient broth. It was then inoculated for $24 \mathrm{~h}$ at $37 \mathrm{deg} \mathrm{C}$. After incubation, culture was further diluted till $10^{5}$ dilution. For inoculation $10^{5}$ dilution was used, the inoculum size was determined with pour plate method, which was approx. $100 \mathrm{cfu} / \mathrm{ml} .0 .2 \mathrm{gm}$ of sample was transferred to $10 \mathrm{ml}$ of Nutrient broth and $100 \mu \mathrm{l}$ of prepared inoculum of E.coli was added to it. Incubation was done for $12 \mathrm{hrs}$ at $37 \mathrm{deg} \mathrm{C}$. After $12 \mathrm{hrs}$ of incubation to check the efficacy, $0.1 \mathrm{ml}$ of the sample was subjected for enumeration. Inoculated $0.1 \mathrm{ml}$ of incubated sample was poured into Petri plate. Pour $12-15 \mathrm{ml}$ of nutrient agar. Incubate at $37{ }^{\circ} \mathrm{C}$ for $24-$ 48 hrs. All colonies were counted in the Petri plate.

\section{Results and Discussion:}

\subsection{Scanning Electron Microscopic Analysis:}

SEM images of neat PLA and four composite samples are shown in Figure 1. Smooth and homogenous surface without any fissures was observed for the neat PLA sheet. SEM images of PLA and PLF composites shows that there was a uniform dispersion of the nano particles up to $10 \mathrm{wt} \%$ of PLF. However, when the concentration of PLF has enhanced to 15 to $20 \mathrm{wt} \%$ one can see visible agglomeration of nano particles in the PLA matrix. 


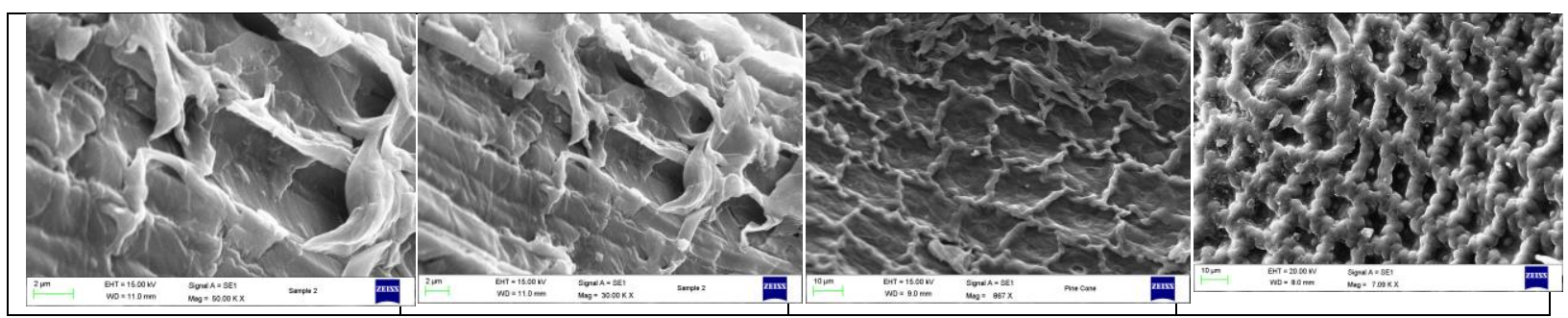

Figure 1. SEM images of neat PLA and its composites

\subsection{XRD analysis:}

Diffractograms of neat PLA and 5-20\% PLF samples are shown in Figure 2. A small peak is observed at $10.8 \mathrm{deg}$, a narrow peak of high intensity around $16.9 \mathrm{deg}$ and a moderate peak at $21.5 \mathrm{deg}$ were observed for neat PLA. These peaks corresponds to the PLA $\alpha-$ crystals. $[2,11]$. PLA and PLF diffractogram peaks remained in the range of $10-23^{\circ}$ as shown by the neat PLA.


Figure 2. Diffractograms of neat PLA and PLF composites

Diffractograms of hybrid composites namely 5\% ZnO/5-20\% PLF/PLA and 10\% $\mathrm{ZnO} / 5-20 \%$ PLF/PLA are shown in Figure 3 and Figure 4. The diffractograms of the composites were almost similar to that of the individual components. For the composite of $5 \% \mathrm{ZnO} / 5 \% \mathrm{PLF} / \mathrm{PLA}$, the intensity of the peak increased when compared to the original PLA. The sharp peaks indicate the more crystalline nature and oriented the composite is. 


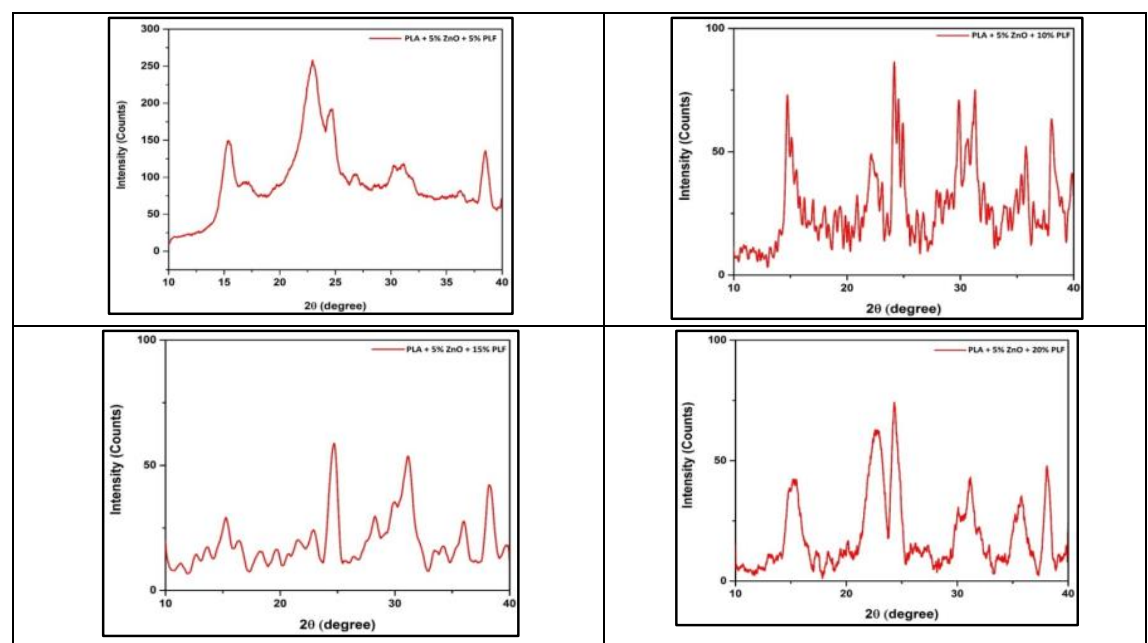

Figure 3. Diffractograms of neat PLA, ZnO and PLF composites

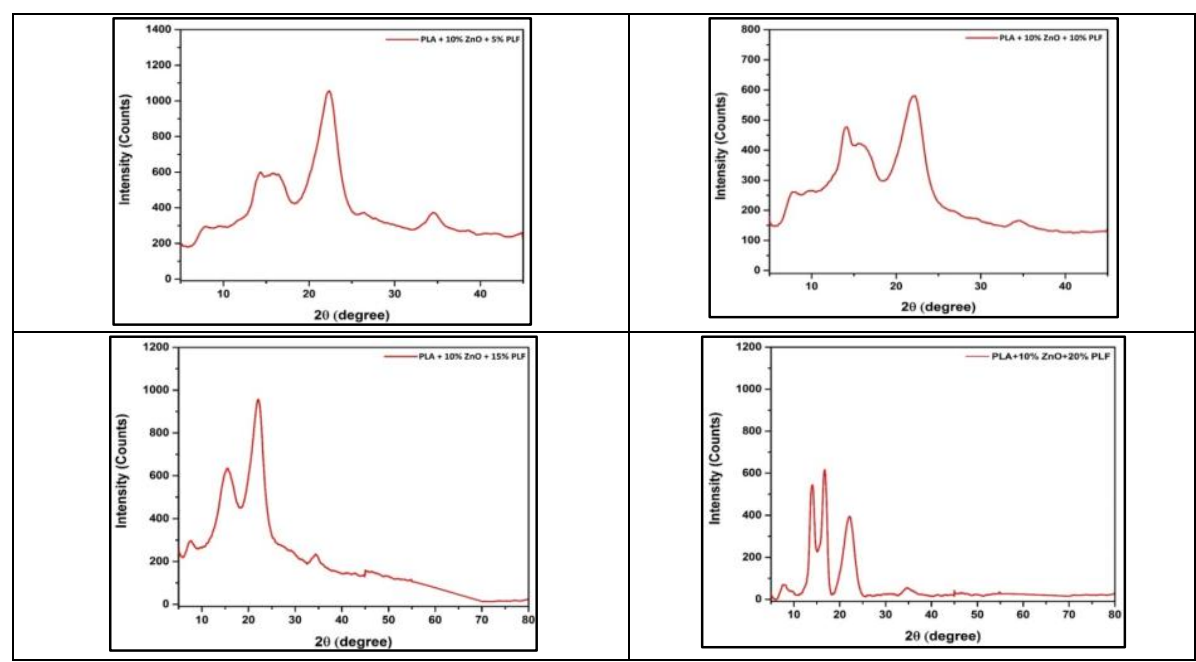

Figure 4. Diffractograms of neat PLA, ZnO and PLF composites

\subsection{Fourier Transform Infrared Spectroscopy (FTIR):}

FTIR spectra of neat PLA and PLA-PLF bio-nanocomposites are depicted in Figure 5. There were noteworthy vibration bands at 3700 to $3200 \mathrm{~cm}^{-1}$ and $2900 \mathrm{~cm}^{-1}$, which are characteristic of the $\mathrm{O}-\mathrm{H}$ stretching vibration and $\mathrm{C}-\mathrm{H}$ stretching, respectively. An exception was the presence of a strong $1749 \mathrm{~cm}^{-1}$ signal, corresponding to the carbonyl $(\mathrm{C}=\mathrm{O})$ stretching peak due to formation of $-\mathrm{COOH}$ groups. The peaks at 1455 and $1375 \mathrm{~cm}^{-1}$ represents the bending vibration of $-\mathrm{C}-\mathrm{H}-$ in $\mathrm{CH}_{3}$ groups. The bands at 1230 and $1110 \mathrm{~cm}^{-1}$ are attributed to the symmetric and asymmetric bending vibrations of C-O-C. The similar bands can be seen in the literature [16]. Amorphous and crystalline phases of PLA were represented by the peaks at 860 and $750 \mathrm{~cm}^{-1}$ respectively.

Figure 6. depicts the FTIR spectrum of the neat PLA $+5 / 10 / 15 / 20 \%$ of PLF. The CNFs from PLF did not interact with the PLA molecules as can be seen from the FTIR spectra of the four samples. Furthermore, because there were no new peaks in all of the PLA- 
PLF bio-nanocomposites, it can therefore be concluded that the incorporation of the PLF was based on a physical rather than chemical interaction.

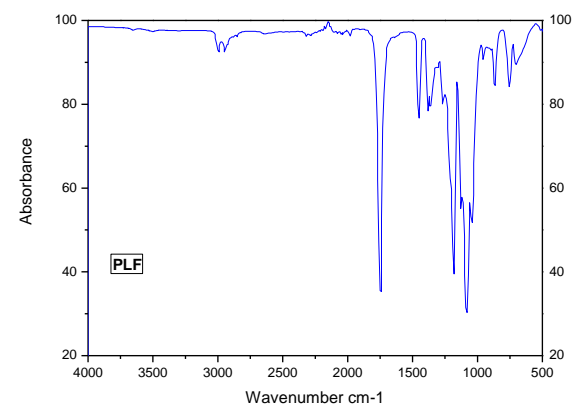

Figure 5. FTIR spectra of the neat PLA

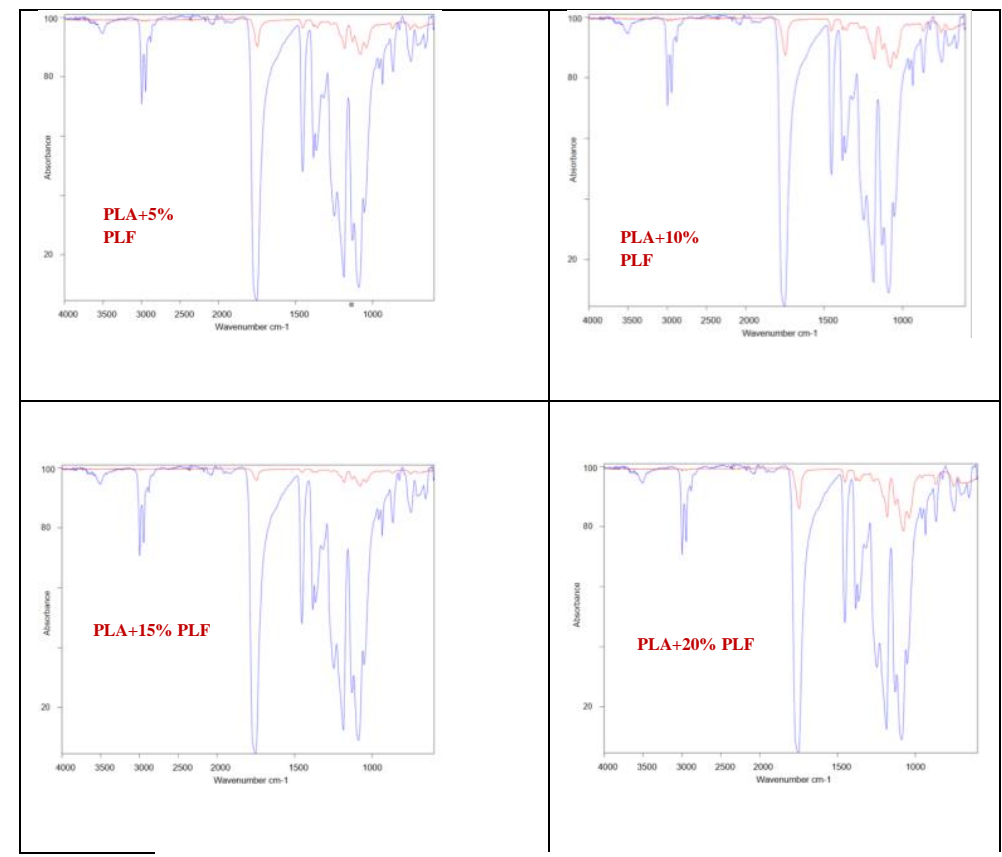

Figure 6. FTIR spectra of the PLA $+5 / 10 / 15 / 20 \%$ of PLF

The Figure 7 shows the spectra of PLA/5\% ZnO with 5/10/15/20 \% PLF composites. All these 4 spectrums resemble that of neat PLA except for the intensity of peaks at various band widths. New peaks around $600-500 \mathrm{~cm}-1$ corresponding to the transverse optical stretching mode of $\mathrm{Zn}-\mathrm{O}$ were noted. Similar trend has been observed for the composites with PLA/10\% ZnO and 5/10/15/20\% PLF (Figure 8). The decrease in the peak intensities of the PLA matrix might be attributed to the masking up by the nano-particles incorporated. 


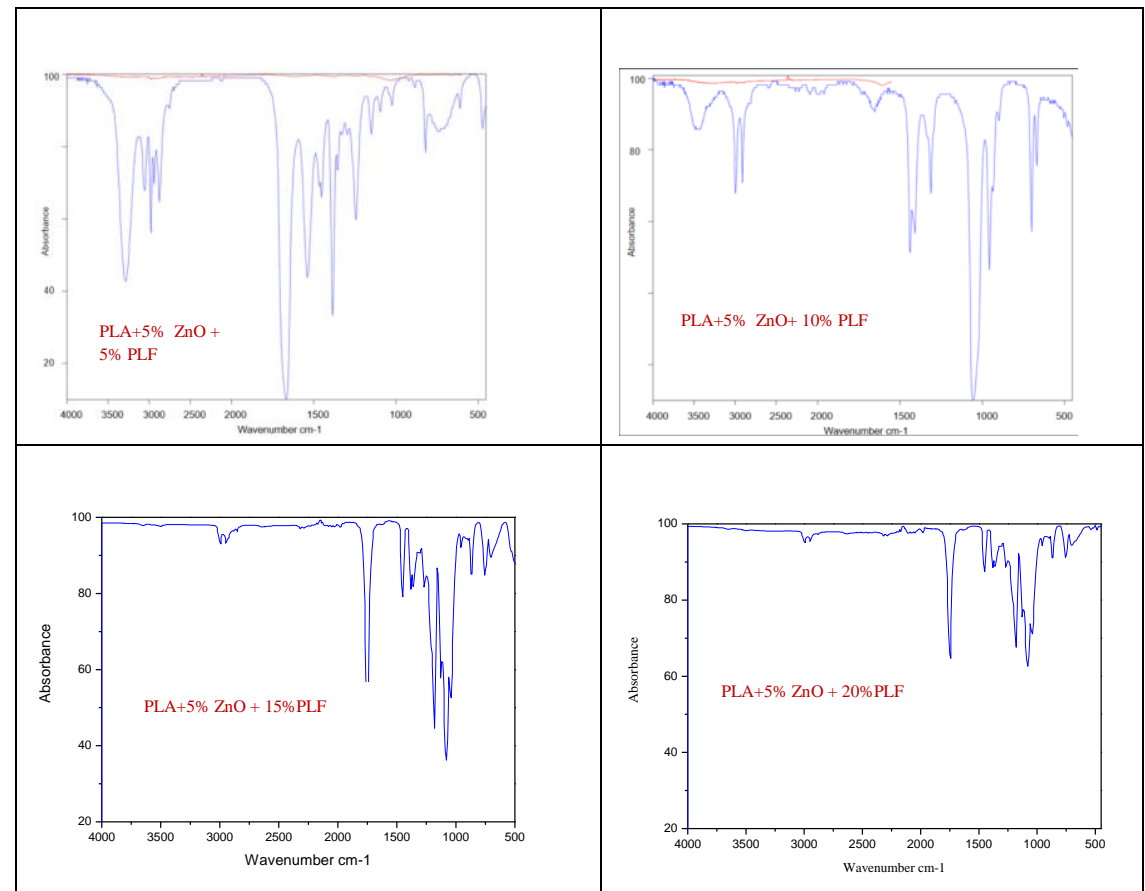

Figure 7. FTIR spectra of the PLA, $5 \%$ ZnO and $5 / 10 / 15 / 20 \%$ of PLF

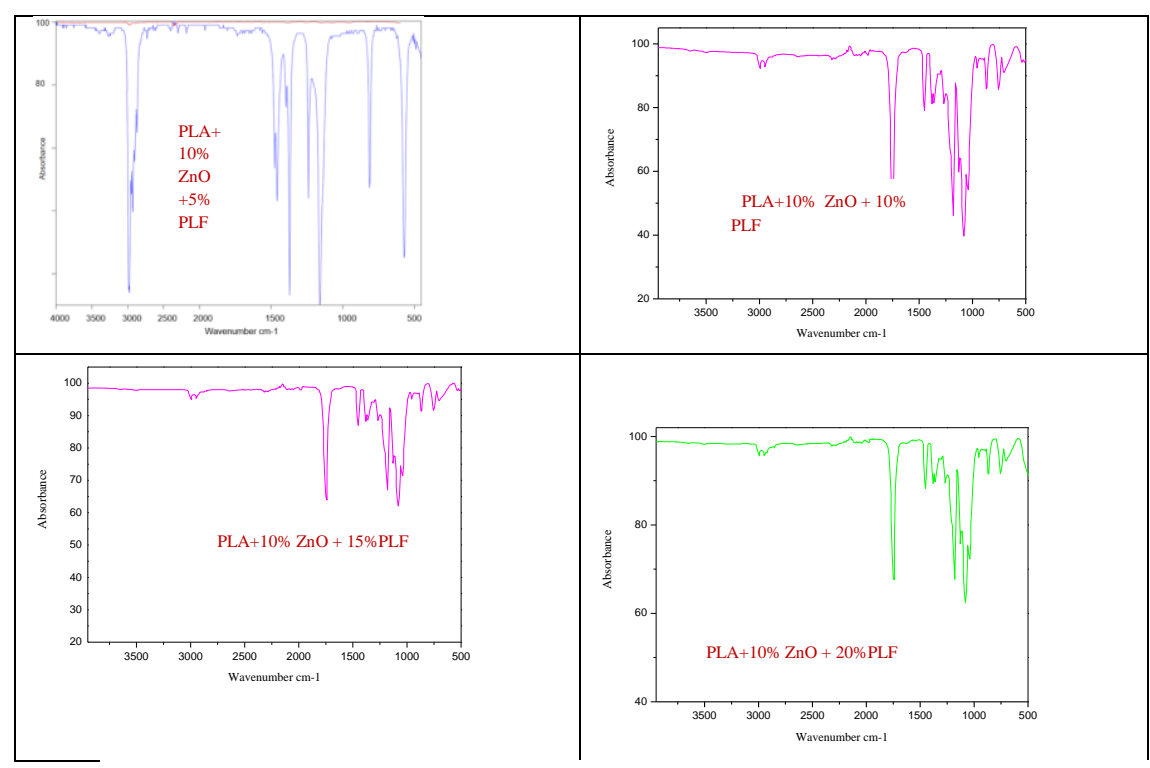

Figure 8. FTIR spectra of the PLA, $10 \% \mathrm{ZnO}$ and 5/10/15/20\% of PLF

\subsection{Thermal analysis of PLA bio composites: Thermogravimetric analysis (TGA):}

$\mathrm{T}_{2}, \mathrm{~T}_{50}, \mathrm{~T}_{90}$ and $\mathrm{T}_{\max }$ ie the temperatures at which $2 \%, 50 \%$ and $90 \%$ of weight of the composites has degraded with the increase in temperature. $\mathrm{T}_{\max }$ is the temperature at which the maximum weight loss has occurred/observed. The values obtained have been tabulated in the Table 1. Thermograms obtained for various compositions may be seen in the Figure 9. 
Table 1. Data obtained from Thermo Gravimetric Analysis (TGA)

\begin{tabular}{|c|l|c|c|c|c|}
\hline S.No & Sample & $\mathbf{T}_{\mathbf{2}}{ }^{\mathbf{}} \mathbf{C}$ & $\mathbf{T}_{\mathbf{5 0}}{ }^{\mathbf{}} \mathbf{C}$ & $\mathbf{T}_{\mathbf{9 0}}{ }^{\mathbf{}} \mathbf{C}$ & $\mathbf{T}_{\max }{ }^{\mathbf{}} \mathbf{C}$ \\
\hline 1 & Neat PLA & 316.72 & 362.36 & 400.36 & 380.62 \\
\hline 2 & PLA + 5 \% PLF & 322.89 & 387.96 & 404.92 & 407.36 \\
\hline 3 & PLA + 10 \% PLF & 323.8 & 387.64 & 405.36 & 407.13 \\
\hline 4 & PLA + 15 \% PLF & 297.32 & 384.52 & 402.17 & 404.15 \\
\hline 5 & PLA + 20 \% PLF & 239.86 & 298.83 & 313.97 & 312.75 \\
\hline 6 & PLA + 5 \% ZnO + 5 \% PLF & 241.12 & 300.16 & 336.79 & 326.39 \\
\hline 7 & PLA + 5 \% ZnO + 10 \% PLF & 242.35 & 301.04 & 361.14 & 338.12 \\
\hline 8 & PLA + 5 \% ZnO + 15 \% PLF & 244.46 & 303.63 & 374.69 & 345.16 \\
\hline 9 & PLA + 5 \% ZnO + 20 \% PLF & 231.97 & 295.96 & 495.68 & 358.09 \\
\hline 10 & PLA + 10 \% ZnO + 5 \% PLF & 231.72 & 293.43 & 496.75 & 345.81 \\
\hline 11 & PLA + 10 \% ZnO + 10 \% PLF & 23.78 & 398.49 & 400.58 \\
\hline 12 & PLA + 10 \% ZnO + 15 \% PLF & 218.56 & 291.88 & 540.81 & 386.5 \\
\hline 13 & PLA + 10 \% ZnO + 20\% PLF & 222.76 & 292.74 & 507.49 & 372.54 \\
\hline
\end{tabular}

Perusal of the thermograms it is evident that the maximum thermal degradation of all the composite samples has occurred in the temperature range of $320-395{ }^{\circ} \mathrm{C}$. The temperature of the maximum rate of degradation ( $\mathrm{T}_{\max }$ ) of the neat PLA was $380.62{ }^{\circ} \mathrm{C}$. The $\mathrm{T}_{\max }$ value of PLA/CNFs binary composite films has shown increased trend up to $407.36{ }^{\circ} \mathrm{C}$.

The $\mathrm{T}_{\max }$ values of PLA/ZnO, PLA/PLF/ZnO hybrid composite films decreased to the low temperature. However, $\mathrm{T}_{\max }$ of PLA $+20 \% \mathrm{ZnO}$ and PLA $+10 \% \mathrm{ZnO}+15 \% \mathrm{PLF}$ were at $408.35{ }^{\circ} \mathrm{C}$ and $386.5{ }^{\circ} \mathrm{C}$ respectively.

With the addition of $\mathrm{ZnO} \mathrm{NPs}(\leq 5 \mathrm{wt} \%)$, the $\mathrm{T}_{\mathrm{g}}, \mathrm{T}_{\mathrm{c}}$ and $\mathrm{T}_{\mathrm{m}}$ values of PLA/ACNC/ZnO composite films further slightly increased due to the inhibition of motion of PLA chains resulted from the well dispersion of $\mathrm{ZnO}$ in composite films. The calorimetric parameters of PLA/ACNC/7\% $\mathrm{ZnO}$ decreased due to the agglomeration of $\mathrm{ZnO}$ [7].

Reduction in thermal stability is directly correlated with the percentage of nanofiller, a higher loading with $\mathrm{ZnO}$ (e.g., 5\%) leading to the most advanced decrease in PLA thermal stability [12]. Abe $\mathrm{H}$ et al [1] had studied thermal degradation of Poly( $\varepsilon$-caprolactone) with addition of Zinc residual compounds and observed the catalytic role of zinc compounds/products at high temperature in promoting PLA degradation leading to the selective formation of lactide and related oligomers. 

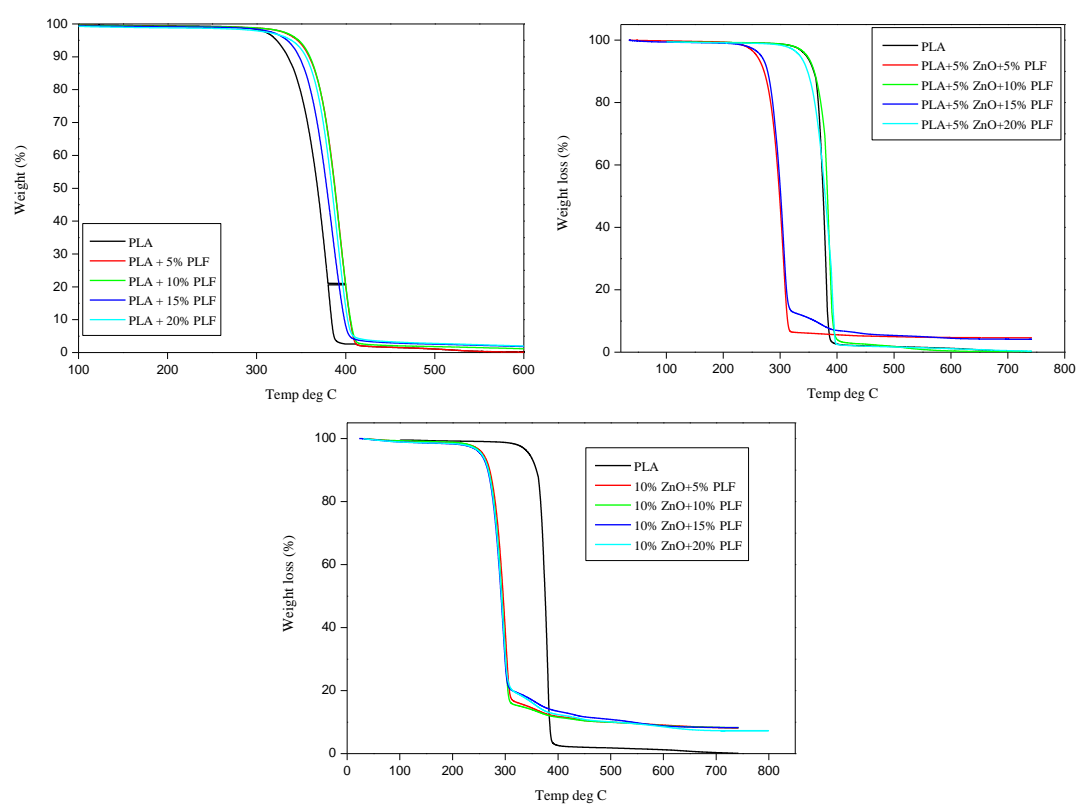

Figure 9. TGA degradation profile of PLA based bio composites

\subsection{Differential Scanning Calorimetry (DSC):}

DSC endotherm of neat PLA and bio composites are shown in Figure 10. The $\mathrm{T}_{\mathrm{g}}$ of neat PLA was $68.59{ }^{\circ} \mathrm{C}$. However, with the incorporation of CNFs from Pineapple leaf fibres, $\mathrm{T}_{\mathrm{g}}$ was observed to decrease and this decline may be due to the aggregation of cellulose nanofibres which were loaded at higher levels. However, PLA with $10 \%$ of PLF has shown $\mathrm{T}_{\mathrm{g}}$ of $96.05^{\circ} \mathrm{C}$ which was different from other compounds. $\mathrm{T}_{\mathrm{cc}}$ and $\mathrm{T}_{\mathrm{m}}$ of neat PLA was 114.53 and $151.72{ }^{\circ} \mathrm{C}$ respectively. Even these temperatures of the PLA and CNF composites decreased slightly for PLF. This phenomenon can be attributed to the source of CNFs synthesized [13, $15]$.

The calorimetric parameters of hybrid composites reveals that $\mathrm{T}_{\mathrm{cc}}$ and $\mathrm{T}_{\mathrm{m}}$ have come down compared to the neat PLA when the $\mathrm{ZnO}$ concentration was kept at $5 \%$ by weight in the matrix. This result may be attributed to the uniform dispersion of $\mathrm{ZnO}$ nano-particles \& PLF in the PLA matrix that might have restricted the mobility of PLA chains, which resulted in the higher absorption of thermal energy. However, when the $\mathrm{ZnO}$ level was enhanced to $10 \%$ by weight, there was no significant change in these calorimetric parameters.
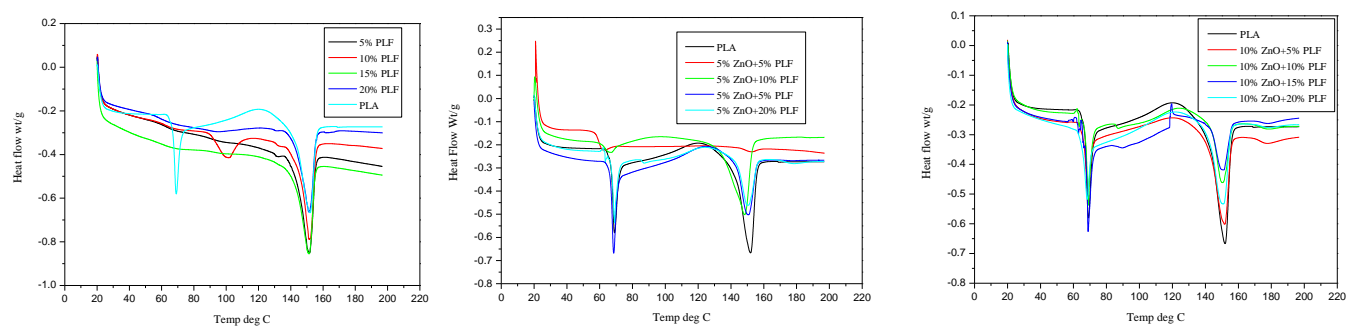

Figure 10. DSC endotherms of PLA based bio composites 


\subsection{Mechanical Properties:}

The results obtained have been tabulated and shown in the Table 2. Analysis of the results obtained reveals that the composite of PLA/10\% PLF has shown maximum tensile strength and Young's Modulus and \% of elongation at break with an increase of $18.8 \%$, $42.39 \%$ and $84.2 \%$ respectively when compared to neat PLA. Detailed examination of the results obtained reveals that the tensile strength, Young's Modulus and percentage elongation at break of the PLA composite with composition of 5\% $\mathrm{ZnO}$ and $10 \% \mathrm{PLF}$ with $5 \% \mathrm{ZnO}$ and PLF composite $35.68 \mathrm{MPa}, 2.74 \mathrm{GPa}$ and $9.24 \%$ respectively. The percentage increase in tensile strength was $27.57 \%$, Young's Modulus was $48.91 \%$ and $\%$ elongation at break has doubled compared to the neat PLA.

Table 2. Mechanical Properties of PLA and its Composites

\begin{tabular}{|c|l|c|c|c|}
\hline S.No. & Sample & $\begin{array}{c}\mathrm{E}_{\mathrm{t}} \\
(\mathrm{GPa})\end{array}$ & $\begin{array}{c}\sigma_{\mathrm{M}} \\
(\mathrm{MPa})\end{array}$ & $\begin{array}{c}\varepsilon_{\mathrm{M}} \\
(\%)\end{array}$ \\
\hline 1 & PLA & 1.84 & 27.92 & 4.96 \\
\hline 2 & PLA +5\%PLF & 2.13 & 30.88 & 6.92 \\
\hline 3 & PLA +10\%PLF & 2.62 & 33.17 & 9.14 \\
\hline 4 & PLA +15\%PLF & 1.92 & 31.26 & 7.40 \\
\hline 5 & PLA +20\%PLF & 1.06 & 24.93 & 5.86 \\
\hline 6 & PLA+5\% ZnO +5\% PLF & 2.28 & 34.74 & 8.06 \\
\hline 7 & PLA+5\% ZnO +10\% PLF & 2.74 & 35.68 & 9.24 \\
\hline 8 & PLA+5\% ZnO +15\% PLF & 1.96 & 30.72 & 7.88 \\
\hline 9 & PLA+5\% ZnO +20\% PLF & 1.52 & 27.16 & 6.65 \\
\hline 10 & PLA+10\% ZnO +5\% PLF & 2.14 & 31.72 & 6.28 \\
\hline 11 & PLA+10\% ZnO +10\% PLF & 2.06 & 29.68 & 8.96 \\
\hline 12 & PLA+10\% ZnO +15\% PLF & 1.84 & 25.16 & 7.15 \\
\hline 13 & PLA+10\% ZnO +20\% PLF & 1.58 & 19.44 & 5.88 \\
\hline
\end{tabular}

\subsection{Bio-degradability:}

The percentage weight loss at various durations was calculated and shown in Table 3. It could be noticed from the table that, with increasing PLF wt. \% in composite, the weight reduction was accelerated gradually. At $20 \mathrm{wt} \%$ of PLF loading to PLA matrix, the percentage of weight loss was observed to be $48.54 \%$. Similar trend has been reported when biodegradability has been studied using soil burial test for PLA-Montmorillonite (MMT) and cellulose nanowhiskers [14]. 
With inclusion of $\mathrm{ZnO}$ to the PLA, the rate of degradation was observed to go down with increase in $\mathrm{ZnO}$ levels. It is also observed that there was no weight loss in the sheet samples from 60 to 120 days. This can be attributed to the anti-microbial resistance of $\mathrm{ZnO}$. The hybrid nano-composite has shown increased bio-degradability rate than the ZnO PLA sheet and nearer to the degradability rate of nono-cellulose sheet. The sample photographs could be seen in Figure 11 to visualise the degradation scheme.

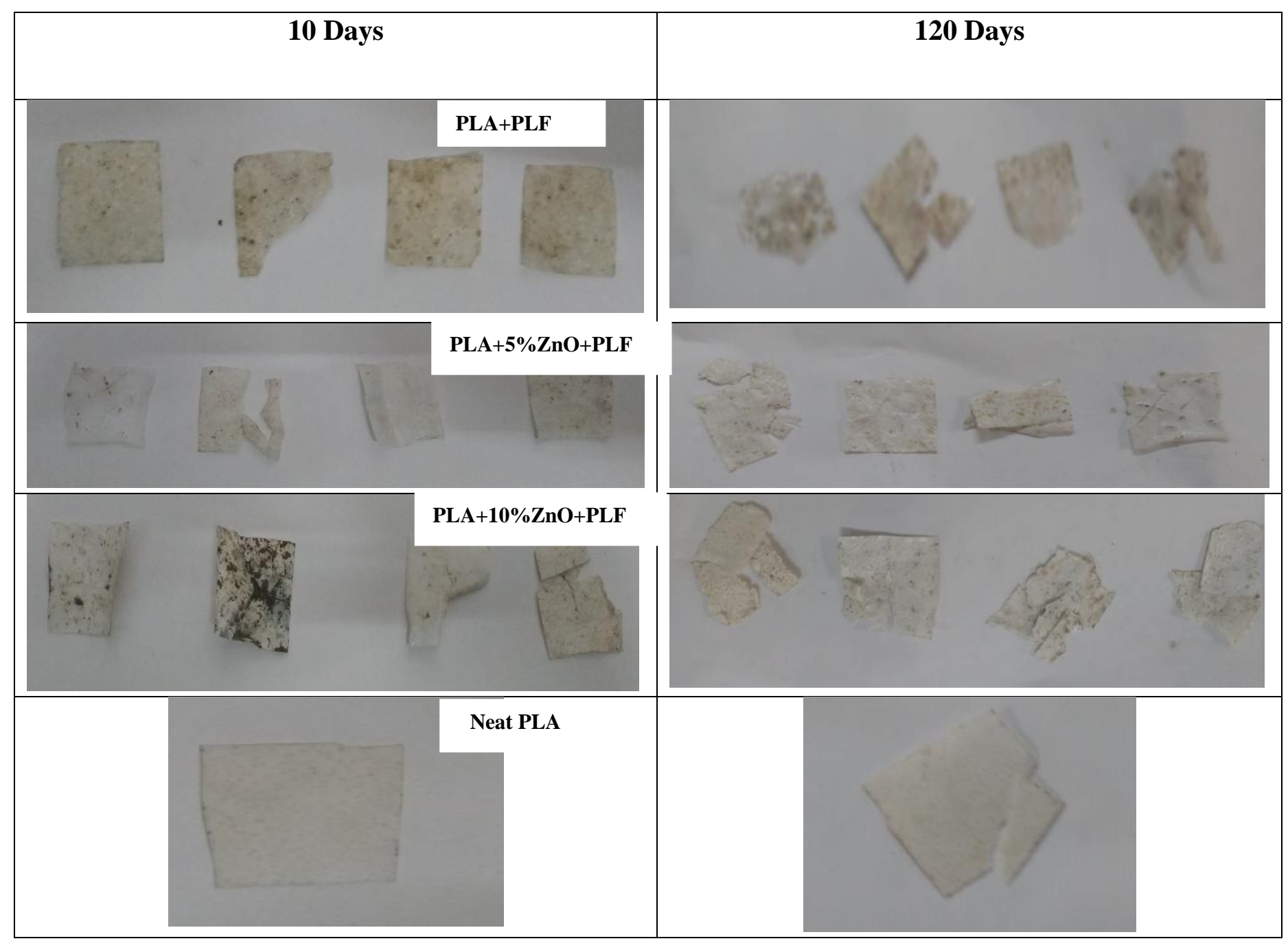

Figure 11. Sample degradation photographs at 10 and 120 days of soil degradation 
Table 3. Weight of the Samples in Grams with age in days and \% weight loss

\begin{tabular}{|c|c|c|c|c|c|c|c|}
\hline \multirow[b]{2}{*}{ S.No. } & \multirow[b]{2}{*}{ Sample } & \multicolumn{5}{|c|}{ Weight (g) } & \multirow{2}{*}{$\begin{array}{l}\% \text { wt. } \\
\text { loss }\end{array}$} \\
\hline & & Day 1 & $\begin{array}{c}\text { Day } \\
10\end{array}$ & $\begin{array}{c}\text { Day } \\
\text { 30 }\end{array}$ & $\begin{array}{c}\text { Day } \\
60\end{array}$ & $\begin{array}{l}\text { Day } \\
120\end{array}$ & \\
\hline 1 & Neat PLA & 0.0961 & 0.0955 & 0.0942 & 0.0883 & 0.0869 & 9.57 \\
\hline 2 & PLA + $5 \%$ PLF & 0.2101 & 0.1963 & 0.1945 & 0.1872 & 0.1753 & 16.56 \\
\hline 3 & PLA + $10 \%$ PLF & 0.1011 & 0.0997 & 0.0876 & 0.0822 & 0.0776 & 23.24 \\
\hline 4 & PLA + $15 \%$ PLF & 0.2267 & 0.2132 & 0.199 & 0.1895 & 0.1503 & 33.7 \\
\hline 5 & PLA + $20 \%$ PLF & 0.1096 & 0.1028 & 0.1012 & 0.0905 & 0.0564 & 48.54 \\
\hline 6 & $\mathrm{PLA}+5 \% \mathrm{ZnO}+5 \% \mathrm{PLF}$ & 0.0408 & 0.0405 & 0.0395 & 0.038 & 0.0337 & 17.41 \\
\hline 7 & $\begin{array}{l}\mathrm{PLA}+5 \% \mathrm{ZnO}+10 \% \\
\mathrm{PLF}\end{array}$ & 0.069 & 0.0682 & 0.0674 & 0.0648 & 0.0557 & 19.27 \\
\hline 8 & $\begin{array}{l}\mathrm{PLA}+5 \% \mathrm{ZnO}+15 \% \\
\mathrm{PLF}\end{array}$ & 0.1131 & 0.1055 & 0.0984 & 0.0961 & 0.0844 & 25.37 \\
\hline 9 & $\begin{array}{l}\mathrm{PLA}+5 \% \mathrm{ZnO}+20 \% \\
\mathrm{PLF}\end{array}$ & 0.0846 & 0.0833 & 0.0721 & 0.0684 & 0.0452 & 46.56 \\
\hline 10 & $\begin{array}{l}\mathrm{PLA}+10 \% \mathrm{ZnO}+5 \% \\
\mathrm{PLF}\end{array}$ & 0.1276 & 0.1252 & 0.109 & 0.1089 & 0.1084 & 15.04 \\
\hline 11 & $\begin{array}{l}\mathrm{PLA}+10 \% \mathrm{ZnO}+10 \% \\
\mathrm{PLF}\end{array}$ & 0.0681 & 0.0677 & 0.0615 & 0.0559 & 0.0538 & 20.99 \\
\hline 12 & $\begin{array}{l}\mathrm{PLA}+10 \% \mathrm{ZnO}+15 \% \\
\mathrm{PLF}\end{array}$ & 0.1693 & 0.1586 & 0.1365 & 0.1318 & 0.1143 & 32.48 \\
\hline 13 & $\begin{array}{l}\mathrm{PLA}+10 \% \mathrm{ZnO}+20 \% \\
\mathrm{PLF}\end{array}$ & 0.1847 & 0.1549 & 0.1464 & 0.1301 & 0.1123 & 39.1 \\
\hline
\end{tabular}

\subsection{Microbial resistance:}

The microbial activity was nonresponsive for only PLA bio composites for both PLA and $\mathrm{ABF}$. With inclusion of $\mathrm{ZnO}$ nanoparticles the microbial resistance /Efficacy (\%R) has gradually increased. With $5 \%$ of $\mathrm{ZnO}$ the $(\% \mathrm{R})$ was obtained $88 \%$ and it increased further to $98 \%$ with $10 \%$. For PLA/ZnO composite with 15 and $20 \%$ of $\mathrm{ZnO}$ the efficacy was found to be $100 \%$. Similar kind of efficacy was noticed with PLA/PLF/ZnO bio composites. The bio composites with almost $100 \%$ (98\%) efficacy was suggesting for sustainable application of the material.

\section{Conclusions:}

The study was focused to produce a bio-degradable composite film using agricultural waste. The PLA produced from simultaneous saccharification and fermentation of starch, the nanocellulose produced from the pineapple leaf fibres has shown improved thermal and mechanical properties up to $10 \%$ of PLF/PLA composite. The microbial resistance of the composites was significant with the addition of nano- $\mathrm{ZnO}$. The biodegradability studies have shown significant reduction in the degradation time of polymer when these nanofibers are 
added. The solvent casted PLA-PLF-ZnO films exhibited multifunctional properties which can be used for environmental friendly applications.

\section{REFERENCES}

1. Abe H, Takahashi N.; Kim K. J, Mochizuki M, Doi Y, Effects of Residual Zinc Compounds and Chain-End Structure on Thermal Degradation of Poly(E-caprolactone), Biomacromolecules, (2004) 5, 1480-1488.

2. Adriana Nicoleta Frone, Denis Mihaela Panaitescu, Ioana Chiulan, Cristian Andi Nicolae, Zina Vuluga, Catalin Vitelaru and Celina Maria Damian, The effect of cellulose nanofibers on the crystallinity and nanostructure of poly(lactic acid) composites, Journal of Material Science, (2016) 51, 9771-9791.

3. Devi LU, Bhagawan SS, Thomas S. Mechanical properties of pineapple leaf fibre-reinforced polyester composites, Journal of Applied Polymer Science, 1997;64:1739-48.

4. Dos Santos Rosa D and Lenz D.M, Biocomposites: Influence of matrix nature and additives on the properties and biodegradation behavior, Biodegradation Engineering Technology, 2013, 433-475.

5. Drzal Lawrence T, Mohanty A and Misra M, Bio-composite materials as alternatives to petroleum-based composites for automotive applications, Magnesium, 2001, 40, 1-3.

6. Farah S, Anderson, D.G, and Langer R, Physical and mechanical properties of PLA, and their functions in widespread applications-A comprehensive review, Adv. Drug Deliv. Rev. 2016, 107, 367-392.

7. Fuyou Yu, Xiang Fei, Yunqing He, Hui Li, Poly(lactic acid)-based composite film reinforced with acetylated cellulose nanocrystals and $\mathrm{ZnO}$ nanoparticles for active food packaging, International Journal of Biological Macromolecules, (2021) 770-779.

8. Graupner, N.; Herrmann, A.S.; Müssig, J. Natural and man-made cellulose fibre-reinforced poly (lactic acid)(PLA) composites: An overview about mechanical characteristics and application areas, Composites. Part A Applied Sciwence Manufacturing, 2009, 40, 810-821.

9. Insoo Kim, Karthika Viswanathan, Gopinath Kasi, Kambiz Sadeghi, Sarinthip Thanakkasaranee and Jongchul Seo, Poly(Lactic Acid)/ZnO Bionanocomposite Films with Positively Charged ZnO as Potential Antimicrobial Food Packaging Materials, Polymers, (2019) 1-17.

10. Kamthai $S$ and Magaraphan $R$, Thermal and mechanical properties of polylactic acid (PLA) and bagasse carboxymethyl cellulose (CMCB) composite by adding isosorbide diesters, AIP Conf. Proc. 2015, 1664.

11. Liqing Wei, Shupin Luo, Armando G. McDonald, Umesh P. Agarwal, Kolby C. Hirth, Laurent M. Matuana, Ronald C. Sabo and Nicole M. Stark, Preparation and Characterization of the Nanocomposites from Chemically Modified Nanocellulose and Poly(lactic acid), Journal of Renewable Materials, (2017) 410-422.

12. Marius Murariu, Yoann Paint, Oltea Murariu, Jean-Marie Raquez, Leila Bonnaud, Philippe Dubois, Current progress in the production of PLA-ZnO nanocomposites: Beneficial effects of chain extender addition on key properties, Journal of Applied Polymer Science, (2015) 1-11.

13. Qianqian Wang, Chencheng Ji, Jianzhong Sun, Qianqian Zhu and Jun Liu, Structure and Properties of Polylactic Acid Biocomposite Films Reinforced with Cellulose Nanofibrils, Molecules, (2020) 1-13.

14. Reza Arjmandi, Azman Hassana, M.K.M. Haafiz, Zainoha Zakaria, Md. Saiful Islam, Structure and Properties of Polylactic Acid Biocomposite Films Reinforced with Cellulose Nanofibrils Effect of hydrolysed cellulose nanowhiskers on properties of montmorillonite/polylactic acid nanocomposites, International Journal of Biological Macromolecules, (2015) 1-13. 
15. Yang Zhangqiang, LI Xiaojie, SI Junhui, CUI Zhixiang, PENG Kaiping, Morphological, Mechanical and Thermal Properties of Poly(lactic acid) (PLA)/Cellulose Nanofibrils (CNF) Composites Nanofiber for Tissue Engineering, Journal of Wuhan University of TechnologyMater. Sci. Ed.,(2019) 207-215.

16. Zhuangzhuang Chu, Tianrui Zhao, Lin Li, Jian Fan and Yuyue Qin, Characterization of Antimicrobial Poly (Lactic Acid)/Nano-Composite Films with Silver and Zinc Oxide Nanoparticles, Materials, (2017) 1-13. 